\title{
Recurrent Primary Hyperparathyroidism: A Diagnostic Challenge in a Supernumerary Intrathyroidal Dormant Adenoma
}

\author{
${ }^{1}$ Aoife Lowery, ${ }^{2}$ Charles Vanbrugghe, ${ }^{3}$ Claudio Golffier, ${ }^{4}$ Frederic Sebag, ${ }^{5}$ Jean Francois Henry
}

\begin{abstract}
Introduction: Recurrent primary hyperparathyroidism is a rare entity and presents a significant surgical challenge.
\end{abstract}

Presentation of case: We present the case of a 53-year-old female with recurrent primary hyperparathyroidism, which was caused by the very rare occurrence of a metachronous parathyroid adenoma in a supernumerary intrathyroidal parathyroid.

Discussion: This unique case highlights the challenges that arise in the evaluation of recurrent hyperparathyroidism in which even a thorough preoperative evaluation including radiographic, nuclear medicine studies and fine needle aspiration cytology did not facilitate a definitive preoperative diagnosis.

Conclusion: The management approach for these patients requires meticulous planning, experience and appropriate use of intraoperative adjuncts to guide surgical strategy.

Keywords: Hyperparathyroidism, Parathyroid, Radiographic study.

How to cite this article: Lowery A, Vanbrugghe C, Golffier C, Sebag F, Henry JF. Recurrent Primary Hyperparathyroidism: A Diagnostic Challenge in a Supernumerary Intrathyroidal Dormant Adenoma. World J Endoc Surg 2015;7(2):39-43.

Source of support: Nil

Conflict of interest: None

\section{CASE REPORT}

A 47-year-old female first presented in 2008 with primary hyperparathyroidism characterized biochemically by hypercalcemia (Ca $2.65 \mathrm{mmol} / \mathrm{l}$ ) and elevated PTH $(120 \mathrm{pg} / \mathrm{ml})$. Cervical ultrasound demonstrated multiple thyroid micronodules with a dominant hypoechoic heterogeneous nodule in the right lobe. An enlarged superior parathyroid was also identified, consistent with a parathyroid adenoma (Fig. 1). Percutaneous fine needle aspiration (FNA) of the dominant thyroid nodule was performed; cytology demonstrated small clusters of follicular cells and sparse colloid consistent with a benign

\footnotetext{
${ }^{1,3}$ Surgical Fellow, ${ }^{2}$ Surgical Resident, ${ }^{4,5}$ Professor

${ }^{1-5}$ Department of Endocrine Surgery, University Hospital Timone, France
}

Corresponding Author: Aoife Lowery, Surgical Fellow, Department of Endocrine Surgery, University Hospital Timone, France Phone: 0033491385854, e-mail: aoife.lowery@gmail.com thyroid nodule. A Technetium $\left({ }^{99 \mathrm{~m}} \mathrm{Tc}\right)$ sestamibi scan exhibited a focus of increased uptake in the middle third of the right thyroid lobe suggestive of a parathyroid adenoma (Fig. 2).

The patient proceeded to a minimally invasive parathyroidectomy via the lateral endoscopic approach. An enlarged right superior parathyroid (P4) was excised and a macroscopically normal right inferior parathyroid (P3) was identified and preserved. Intraoperative parathyroid hormone (PTH) assay confirmed a $>50 \%$ drop in circulating PTH from 143 to $21 \mathrm{pg} / \mathrm{ml}$ post excision of the superior parathyroid and pathological examination

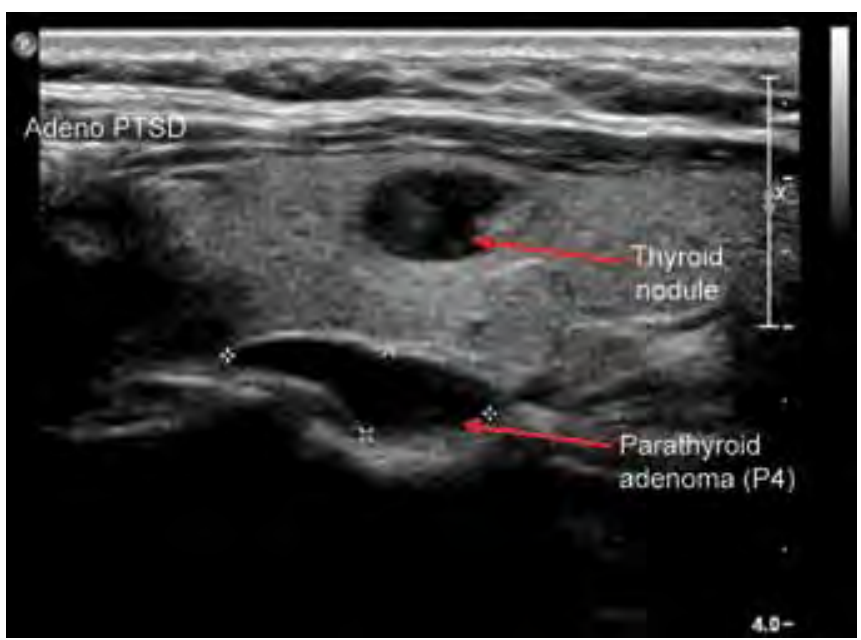

Fig. 1: Thyroid and parathyroid ultrasound prior to the first parathyroidectomy in 2008 clearly demonstrating a posteriorly located parathyroid adenoma and an intrathyroid lesion

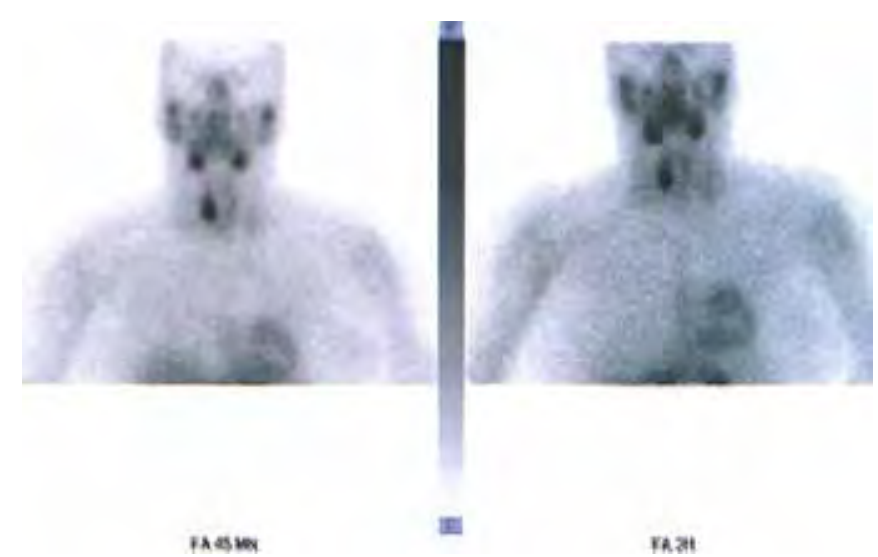

Fig. 2: Technetium ${ }^{99 \mathrm{~m}} \mathrm{Tc}$ ) sestamibi scan prior to the first parathyroidectomy exhibiting a focus of increased uptake in the middle third of the right thyroid lobe suggestive of a parathyroid adenoma 
confirmed a parathyroid adenoma $(18 \times 10 \times 4 \mathrm{~mm}, 68 \mathrm{mg})$. Postoperative calcium and PTH were normal and the patient remained asymptomatic until she represented in 2013 with a biochemical profile consistent with recurrent hyperparathyroidism (Graph 1). Technetium ${ }^{99 \mathrm{~m}} \mathrm{Tc}$ ) sestamibi scan revealed persistent hyperfixation in the mid-superior aspect of the right thyroid lobe corresponding to an intrathyroidal lesion, unchanged since the previous exam in 2008 (Fig. 3). Ultrasound (US) identified a multinodular thyroid gland with a macronodule corresponding to the area of increased uptake on scintigraphy (Fig. 4). Fine needle aspiration of the dominant thyroid nodule was repeated and cytology demonstrated regular follicular thyroid cells, macrophages and colloid, classified as Bethesda 2.

The patient proceeded to bilateral cervical exploration, which identified a macroscopically normal left superior parathyroid (P4), and a macroscopically enlarged left inferior parathyroid (P3), which was excised and subsequently proven to be normal on pathological analysis. Intraoperative PTH assay did not confirm a sufficient drop of $50 \%$ in circulating PTH following excision (94.3-69.8 pg/ml). Exploration of the right-side identified no pathologic parathyroid, however a nodule was palpated in the thyroid lobe, corresponding to the area of increased uptake on scintigraphy. It was decided to perform a right thyroid lobectomy due to the large size of this intrathyroidal lesion, after which circulating PTH dropped by $>50 \%$ to $13.02 \mathrm{pg} / \mathrm{ml}$. Examination of the excised right thyroid lobe revealed an intrathyroidal parathyroid adenoma (Fig. 5). This was confirmed histologically and the patient exhibited biochemical cure with a serum calcium of $2.45 \mathrm{mmol} / \mathrm{l}$ and PTH $13.2 \mathrm{pg} / \mathrm{ml}$ at postoperative follow-up.

\section{DISCUSSION}

Recurrent primary hyperparathyroidism (PHPT) is rare and refers to the reappearance of hypercalcemia after at least 6 months of normocalcemia, implying that the patient has been cured during the interval between the first operation and the reappearance of hypercalcemia. Our patient was normocalcemic for almost 5 years after the initial parathyroidectomy (Fig. 3). Recurrent disease may develop in residual glands that were normal at the time of initial exploration (metachronous adenoma), recurrence at the site of previous resection/autotransplantation, local recurrence/distant metastases after excision of a parathyroid carcinoma or rare cases of parathyromatosis.

It has been suggested that the minimally invasive approach to parathyroidectomy may result in increased risk of persistent and recurrent hyperparathyroidism as all four glands are not visualized. ${ }^{1,2}$ However, four gland exploration as the initial approach in our patient is unlikely to have changed management as two glands were identified on the right-side and the pathologic gland was correctly excised at the first operation leading to a biochemical cure. The pathologic gland responsible for the recurrence of hyperparathyroidism was both intrathyroidal and supernumerary and was not hypersecreting at the time of the initial operation, evidenced by the persistent drop in PTH postoperatively.

In this case, the search for the pathologic gland was complicated by the fact that this gland was both supernumerary and ectopic (intrathyroidal). A supernumerary intrathyroidal gland is a very rare anomaly, found in only $0.5 \%$ of reported autopsy series. ${ }^{3}$ In patients undergoing cervical exploration for hyperparathyroidism the reported incidence of an intrathyroidal parathyroid

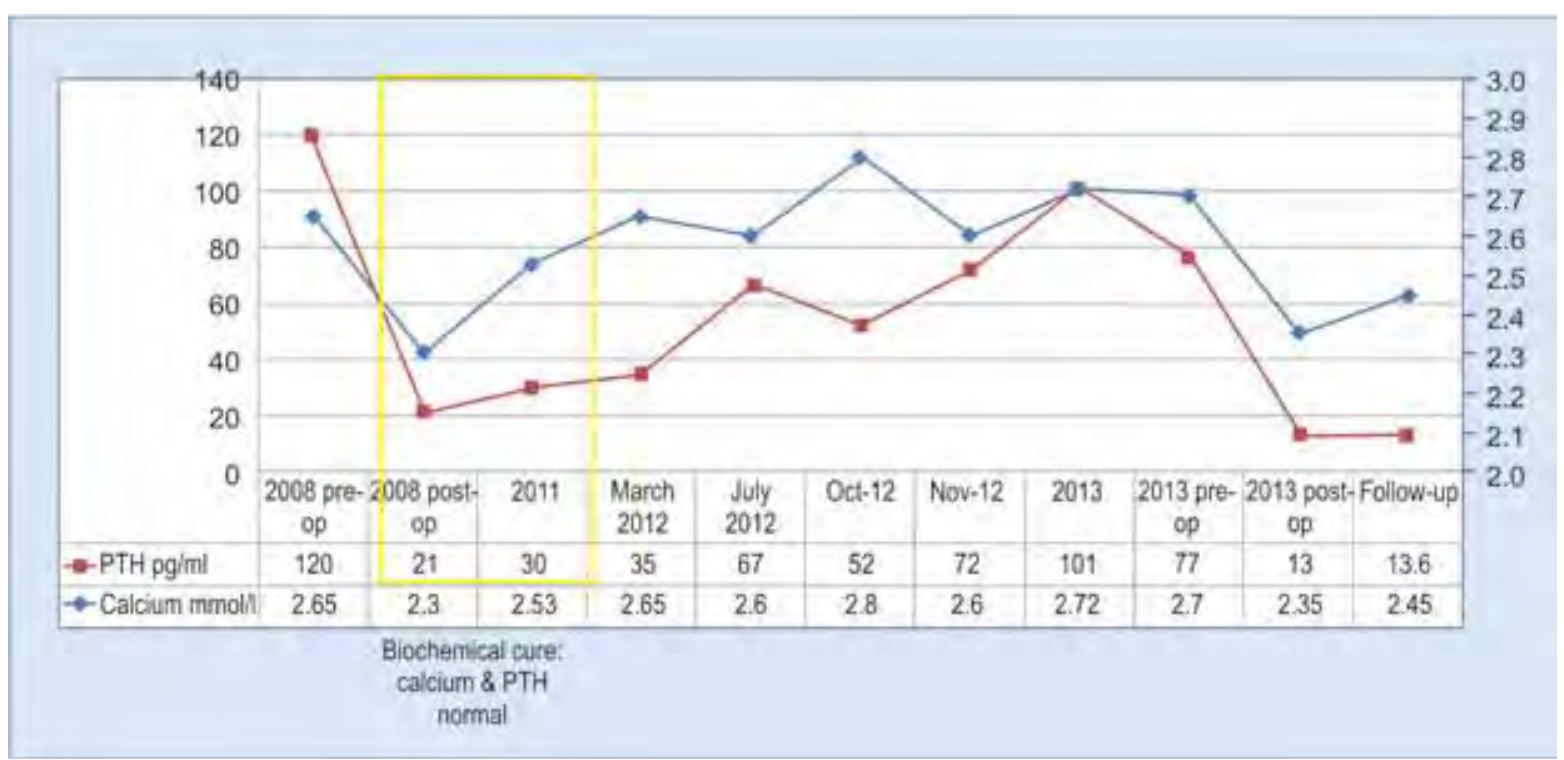

Graph 1: Biochemical profile from 2008 to 2013. The calcium and PTH remained normal between 2008 and 2012 confirming a biochemical cure 


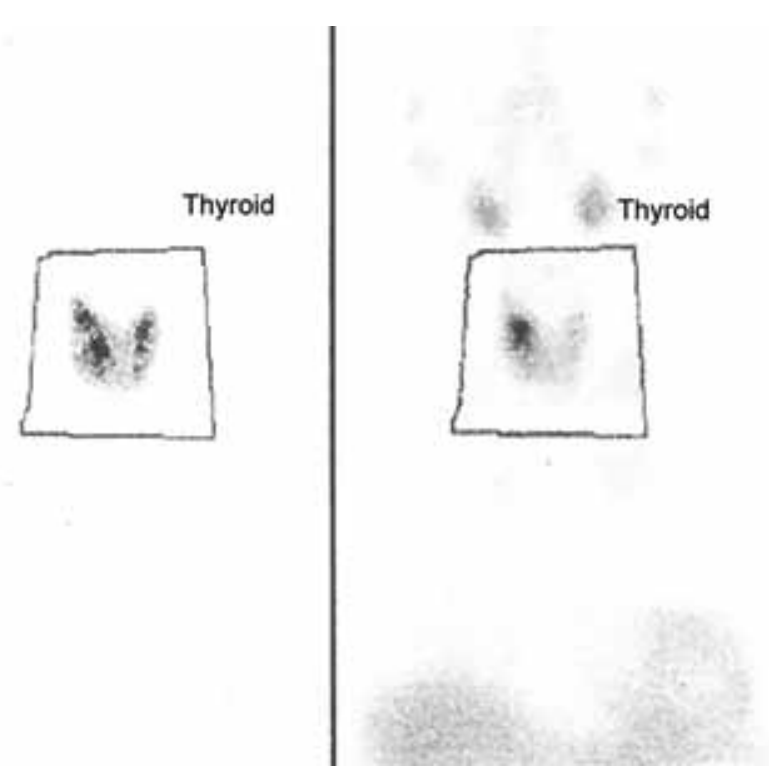

Fig. 3: Technetium $\left({ }^{99 \mathrm{~m}} \mathrm{Tc}\right)$ sestamibi scan performed prior to second surgery showing persistent hyperfixation in the midsuperior aspect of the right thyroid lobe corresponding to an intrathyroidal lesion, unchanged since the previous exam in 2008

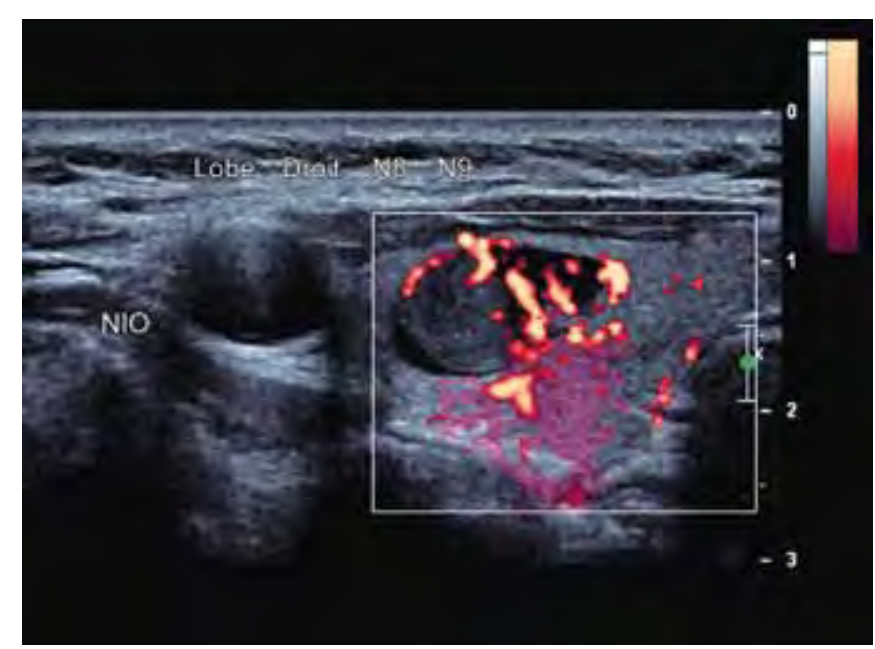

Fig. 4: Thyroid and parathyroid ultrasound prior to the second cervical exploration demonstrating a well vascularized lesion in the right thyroid lobe

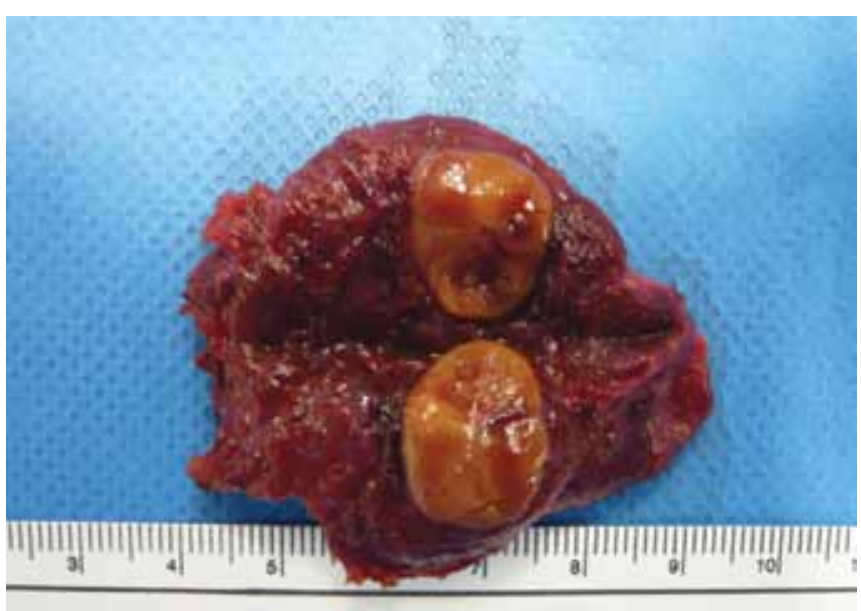

Fig. 5: Gross pathology of the cut thyroid lobe demonstrating an intrathyroidal parathyroid adenoma occupying a large proportion of the thyroid lobe, thus necessitating thyroid lobectomy ranges from 0.7 to $3.6 \%$, depending on the series and the definition of and 'intrathyroidal' lesion used. ${ }^{4-6}$ Consistently higher rates are reported in patients undergoing reoperative parathyroid surgery. ${ }^{7-9}$

A thorough knowledge of anatomy and an understanding of the embryonic development of the parathyroids is critical to successful localization of ectopic glands. The truly intrathyroidal parathyroid is a result of congenital ectopia; the parathyroids originate from the 3rd and 4th branchial pouches and migrate caudally to their final positions. The embryologic migration of the superior parathyroids (P4) occurs simultaneously with the ultimobranchial bodies and a P4 may become included within the thyroid during fusion of the ultimobranchial bodies with the median thyroid rudiment creating an intrathyroidal parathyroid, surrounded on all sides by thyroid tissue. The location of the parathyroid adenoma in this case supports this embryological origin. Furthermore, the second adenoma in this case developed in a supernumerary intrathyroidal gland; supernumerary glands arise from fragmentation of normal parathyroid tissue, when the pharyngeal pouches separate from the pharynx and can occur either adjacent to the gland of origin or in an ectopic location, the supernumerary intrathyroidal parathyroid in this instance may have fragmented from the superior parathyroid/P4 which was excised at the first operation.

The presence of a supernumerary intrathyroidal gland, while rare, has important management implications. Preoperative localization with sestamibi and ultrasound (US) is essential. These imaging techniques correctly identify $95 \%$ of abnormal parathyroid glands. ${ }^{10}$ For intrathyroidal parathyroids specifically, US +/-color Doppler may be the most informative imaging method. ${ }^{5,11}$ Heller et al reported a sensitivity and specificity for detecting intrathyroid parathyroid adenomas of 76 and $92 \%$, using ultrasound criteria including solid composition, hypoechogenicity and presence of a feeding polar vessel. The sensitivity and specificity for differentiating an intrathyroid parathyroid adenoma from a thyroid nodule were 78 and $86 \%$ respectively. ${ }^{12}$ The presence of a multinodular goiter limits the accuracy of both imaging modalities. The ultrasound in this case demonstrated a multinodular thyroid with a richly vascularized dominant nodule in the right lobe (Graph 1). Fine needle aspiration cytology however, both in 2008 and 2013 contained colloid and follicular thyroid cells, leading to the conclusion that this was a benign thyroid nodule. The value of cytology in the diagnosis of thyroid nodules is well accepted. However, intrathyroidal parathyroid adenoma is a potential diagnostic pitfall of this test, because it can resemble a thyroid lesion. The problem is most frequently encountered when 
the cells are oncocytic due to the similar morphology of oncocytic lesions of the thyroid and parathyroid. ${ }^{13-15}$ In this case, however the lesion was not oncocytic and the presence of thyroid follicular cells in the FNA specimen may be explained by the truly intrathyroidal nature of this parathyroid adenoma. Many so-called intrathyroid adenomas are embedded deep in a crevice of the thyroid parenchyma or hidden just under the thyroid capsule, in this instance however, the parathyroid was located deep within the thyroid parenchyma (Fig. 3) so it is conceivable that the FNA needle traversing the thyroid parenchyma may have been responsible for the presence of colloid and thyroid follicular cells that led to the misleading report of a benign thyroid nodule. In this case, immunocytological staining of the aspirated cells with parathormone and thyroglobulin antibodies was not performed, which could have differentiated a thyroid from a parathyroid lesion. Indeed, a parathormone assay of the material obtained by FNA may have been more informative than cytology in this instance.

In the management of recurrent HPT, it is critical to confirm the diagnosis and plan the operative strategy prior to reoperation. A thorough review of all previous data including imaging, operative notes and illustrations, intraoperative and postoperative PTH and pathology reports is essential for the surgeon to gain an impression of whether the patient has inadequately treated multigland disease or an occult/metachronous parathyroid adenoma. Based on a review of this data, the surgeon plans the reoperative strategy. If an adenoma is suspected and well localized on preoperative imaging, it is reasonable to employ a minimally invasive approach with the use of intraoperative PTH monitoring. Where multigland disease is suspected, the cervical exploration should include meticulous bilateral exploration to identify and assess all glands. This also involves a search for supernumerary glands and a bilateral thymectomy if necessary. Intraoperative PTH monitoring proved invaluable in this case; the insufficient drop in PTH following excision of a macroscopically enlarged left inferior parathyroid prompted continued exploration and ultimately a thyroid lobectomy and confirmation of an intrathyroidal parathyroid adenoma. It is important to note that thyroid excision is rarely necessary; ${ }^{16}$ as the parathyroid may lie in a deep thyroid sulcus rather than a truly intrathyroidal location, the capsule should be explored thoroughly before considering thyroid excision. ${ }^{3}$ There remains a role for thyroid excision as the last available procedure, when a complete cervical exploration has failed to yield the pathologic gland, but this is only indicated when preoperative investigations or digital exploration suggest an intrathyroid location. ${ }^{17}$
The nature of an intrathyroidal lesion can also be further characterized by needle aspiration and parathormone assay of the material obtained, this approach has been shown to have a high sensitivity $(88 \%)$ and positive predictive value $(100 \%)$ for identification of parathyroid adenomas in the preoperative setting ${ }^{18}$ and can be translated effectively to the intraoperative setting using an intraoperative PTH assay. ${ }^{19}$

The surgeon may also consider the use of intraoperative ultrasound to precisely locate the intrathyroidal parathyroid $^{20}$ and enucleation of an intrathyroidal parathyroid can be performed if the size and location of the parathyroid adenoma are favorable to allow this procedure without compromising the integrity of the thyroid lobe.

This case highlights the challenge of managing recurrent hyperparathyroidism in the very rare setting of a supernumerary intrathyroidal pathologic gland. In such cases, the experienced surgeon must rely on an understanding of specific anatomy and embryology; careful preparation and meticulous cervical exploration with the benefit of intraoperative adjuncts is likely to be the most successful approach.

\section{REFERENCES}

1. Schneider DF, Mazeh H, Sippel RS, Chen H. Is minimally invasive parathyroidectomy associated with greater recurrence compared to bilateral exploration? Analysis of more than 1,000 cases. Surgery 2012;152(6):1008-1015.

2. Norman J, Lopez J, Politz D. Abandoning unilateral parathyroidectomy: why we reversed our position after 15,000 parathyroid operations. J Am Coll Surg 2012;214(3):260-269.

3. Akerstrom G, Malmaeus J, Bergstrom R. Surgical anatomy of human parathyroid glands. Surgery 1984 Jan;95(1):14-21.

4. Feliciano DV. Parathyroid pathology in an intrathyroidal position. Am J Surg 1992 Nov;164(5):496-500.

5. Proye C, Bizard JP, Carnaille B, Wuievreux JL. Hyperparathyroidism and intrathyroid parathyroid gland. 43 cases. Ann Chir 1994;48(6):501-506.

6. Goodman A, Politz D, Lopez J, Norman J. Intrathyroid parathyroid adenoma: incidence and location-the case against thyroid lobectomy. Otolaryngol Head Neck Surg 2011;144(6): 867-871.

7. Gough I. Reoperative parathyroid surgery: the importance of ectopic location and multigland disease. ANZ J Surg 2006 Dec;76(12):1048-1050.

8. Mariette C, Pellissier L, Combemale F, Quievreux JL, Carnaille B, Proye C. Reoperation for persistent or recurrent primary hyperparathyroidism. Langenbecks Arch Surg 1998; 383(2):174-179.

9. Shen W, Düren M, Morita E, Higgins C, Duh QY, Siperstein AE, Clark $\mathrm{OH}$. Reoperation for persistent or recurrent primary hyperparathyroidism. Arch Surg 1996;131(8):861-867.

10. Henry JF. Reoperation for primary hyperparathyroidism: Tips and tricks. Langenbecks Arch Surg 2010 Feb;395(2):103-109.

11. Abboud B, Sleilaty G, Ayoub S, et al. Intrathyroid parathyroid adenoma in primary hyperparathyroidism: can it be predicted preoperatively? World J Surg 2007 Arp;31(4):817-823. 
12. Heller MT, Yip L, Tublin ME. Sonography of intrathyroid parathyroid adenomas: are there distinctive features that allow for preoperative identification? Eur J Radiol 2013; 82(1):e22-27.

13. Paker I, Yilmazer D, Yandakci K, Arikok AT, Alper M. Intrathyroidal oncocytic parathyroid adenoma: a diagnostic pitfall on fine needle aspiration. Diag Cytopath 38(11): 833-836.

14. Dimashkieh $\mathrm{H}$, Krishnamurthy S. Ultrasound guided fine needle aspiration biopsy of parathyroid gland and lesions. Cyto J 2006;3:6.

15. Absher KJ, Truong LD, Khurana KK, Ramzy I. Parathyroid cytology: avoiding diagnostic pitfalls. Head Neck 2002;24:157-164.

16. Goodman A, Politz D, Lopez J, Norman J. Intrathyroid parathyroid adenoma: incidence and location-the case against thyroid lobectomy. Otolaryngol Head Neck Surg 2011;144(6):867-871.
17. Herden U, Seiler CA, Candinas D, Schmid SW. Intrathyroid adenomas in primary hyperparathyroidism: are they frequent enough to guide surgical strategy? Surg Innov 2011;18(4):373-378.

18. Dutta D, Maisnam I, Selvan C, Ghosh S, Mukhopadhyay S, Chowdhury S. Role of parathyroid hormone estimation in needle washing of parathyroid aspiration biopsy in localizing 99m Tc-sestamibi negative primary hyperparathyroidism: a series of seventeen patients. Clin Otolaryngol 2014 Apr 29. DOI: 10.1111/coa.12248.

19. Pelizzo MR, Losi A, Boschin IM, Toniato A, Pennelli G, Sorgato N, Faggian D, Plebani M. Rapid intraoperative parathyroid hormone assay in fine needle aspiration for differential diagnosis in thyroid and parathyroid surgery. Clin Chem Lab Med 2010 Sep;48(9):1313-1317.

20. Sopiński J, Kuzdak K. Navigation with use of intraoperative ultrasound in search for neoplastic lesions of endocrine glands. Pol Przegl Chir 2013;1;85(5):262-270. 